\title{
Histological diversity and molecular characteristics in gastric cancer: relation of cancer stem cell-related molecules and receptor tyrosine kinase molecules to mixed histological type and more histological patterns
}

\author{
Kazuhiro Sentani ${ }^{1}$ (1) $\cdot$ Takeharu Imai $^{1} \cdot$ Go Kobayashi ${ }^{1} \cdot$ Tetsutaro Hayashi $^{1} \cdot$ Naomi Sasaki $^{2} \cdot$ Naohide Oue $^{1}$. \\ Wataru Yasui ${ }^{1}$
}

Received: 18 August 2020 / Accepted: 28 September 2020 / Published online: 28 October 2020

(c) The International Gastric Cancer Association and The Japanese Gastric Cancer Association 2020

\begin{abstract}
Background Gastric cancers (GCs) are still one of the leading causes of cancer-related mortality. The histological and molecular features of GC may differ widely from area to area within the same tumor. Intratumoral heterogeneity has been considered a major obstacle to an efficient diagnosis and successful molecular treatment.

Methods We selected and reevaluated $842 \mathrm{GC}$ cases and analyzed the relationship between numbers or composites of histological patterns within tumors, and clinicopathological parameters in mucosal and invasive areas. In addition, we searched for the GC-associated molecules or molecular subtypes marking histological diversities.

Results GC cases with more histological numbers or mixed types in invasive areas showed significantly higher $\mathrm{T}$ grade and staging, whereas those in mucosal areas did not show any significant associations. GCs with histological diversities showed poorer prognosis and characteristically expressed cancer stem cell-related molecules (CD44, CD133 or ALDH1) and receptor tyrosine kinase molecules (HER2, EGFR or c-MET) as well as Helicobacter pylori infection. Expressions of CD44, HER2, c-MET, laminin $5 \cdot 2$ or retained E-cadherin in mucosal areas were predictive of more histological numbers and mixed types in invasive areas. In addition, the chromosomal instability subtype of GC showed significant associations with more histological numbers and mixed histological type, whereas the genomic stability subtype of GC showed a significant relationship with pure type.

Conclusions We displayed the relationship between histological diversity and molecular features in GC, and we hope that the present data can contribute to the early diagnosis and prevention, and effective treatment of GC.
\end{abstract}

Keywords Gastric cancer $\cdot$ Histological diversity $\cdot$ Molecular characteristic $\cdot$ Stem cell-related molecule $\cdot$ Receptor tyrosine kinase molecule

Electronic supplementary material The online version of this article (https://doi.org/10.1007/s10120-020-01133-w) contains supplementary material, which is available to authorized users.

Kazuhiro Sentani

kzsentani@hiroshima-u.ac.jp

1 Department of Molecular Pathology, Graduate School of Biomedical and Health Sciences, 1-2-3 Kasumi, Minami-ku, Hiroshima 734-8551, Japan

2 Department of Pathology, Kure-Kyosai Hospital, Federation of National Public Service Personnel Mutual Aid Associations, Hiroshima, Japan

\section{Introduction}

Gastric cancers (GCs) are still one of the leading causes of cancer-related mortality. The high mortality of GCs is mainly due to late diagnosis and poor response to the currently available therapeutic agents. One of the reasons that may explain poor clinical outcomes of GCs is their highly heterogeneous nature. Unlike other tumors of the digestive system, GCs exhibit diversity in various pathological factors, including histological type, differentiation, stroma and infiltration patterns. Histological type is one of the most important factors, because it has a close relationship to the aggressiveness of the disease or prognosis of patients with 
GCs [1]. It is known that patients with poorly differentiatedtype GCs have a worse prognosis than those with well-differentiated-type GCs [2]. The histological features of GCs vary from lesion to lesion and within the same tumor due to heterogeneity. Even tumors confined to the mucosa show histologic diversity, which tends to increase with deeper invasion and increased tumor diameter [3]. As mucosal tumors grow or invade into submucosal or deeper layers, the numbers or composites of histological patterns are likely to change [4]. Differentiated-type GCs were reported to progress to undifferentiated type [5]. Mixed-type GCs are defined as differentiated-type cancers containing undifferentiated-type components or undifferentiated-type cancers containing differentiated-type components. Current guidelines stipulate that mixed-type cancers of predominantly the differentiated type are to be dealt with as differentiated-type cancers and those of predominantly the undifferentiated type as undifferentiated type. Takizawa et al. examined surgical specimens of mixed histological-type GCs and found that the frequency of lymphovascular invasion and risk of lymph node metastasis was higher in mixed-type GC than in pure differentiated-type GC, which indicates the higher malignancy of mixed type [6].

Although the biological characteristics correlate with morphological diversities during GC progression [3, 7], histological classifications are not always sufficient to explain the high complexity of GCs. Tumor heterogeneity can also be generated due to genetic alterations, epigenetic abnormalities, interactions between tumor cells and the microenvironment, and interactions between different tumor cell populations [8]. In recent years, there has been outstanding progress in the elucidation of the genomic landscape of GCs due to technical advances and the efforts of international research consortiums such as The Cancer Genome Atlas (TCGA) Research Network [9]. The identification of different molecular subtypes of GCs represents an advance towards the goal of personalized medicine. Nevertheless, in current clinical practice, the choice of therapeutic strategy against GCs still does not regularly consider molecular heterogeneity and is mostly based on tumor stage. Considering the well-known intratumoral heterogeneity in GCs, random tissue submitted for genomic analysis may not fully represent the underlying mechanisms of the whole tumor. Thus, the combined molecular and histological classification defines the pathogenetic mechanism of GCs more accurately. In addition, we raised questions about what molecules would be associated with histological diversities in invasive areas and whether histological features in invasive areas could be predicted preoperatively by mucosal histology or molecular characteristics.

In the present study, we categorized each of the GCs into a mucosal component and invasive component, analyzed the relationship of numbers of histological patterns and composites with clinicopathological parameters and prognosis, and finally searched for GC-associated molecules marking histological diversities.

\section{Materials and methods}

\section{Patients and histological evaluation}

At first, we collected archival formalin-fixed paraffin-embedded tissues from 1067 consecutive cases who had undergone surgical excision for GC at Hiroshima University Hospital or its affiliated hospitals. 842 GC cases (539 men and 303 women; age range, 31-93 years) were pathologically proven invasive GC with mucosal components, and such cases showing mucosal invasion by retrograde spread from underneath and by lymphatic or vascular permeation were excluded from the present study (Supplementary Fig. 1a-d). All 842 GC cases were histologically reviewed and reclassified by two pathologists (K. S. and W. Y.) based on the latest 15th edition of the Japanese Classification of Gastric Carcinomas. Histological numbers are defined as the numbers of histological patterns within the tumors. The concordance rate for diagnosis between the two pathologists was more than $80 \%$, and when the evaluations differed, a final diagnosis was made by consensus, while the investigators reviewed the specimen with a multi-head microscope. Tumor staging was classified according to the latest 8th edition of the AJCC/UICC TNM classification of malignant tumors. 124 out of $842 \mathrm{GC}$ cases were used for immunohistochemical analysis because of the availability and reliability of their prognostic information. Because written informed consent was not obtained from the patients, identifying information for all samples was removed before analysis to ensure strict privacy protection. This procedure was in accordance with the Ethical Guidelines for Human Genome/Gene Research enacted by the Japanese Government. This study was approved by the Ethical Committee for Human Genome Research of Hiroshima University, Hiroshima, Japan (No. IRINHI66).

\section{Immunohistochemistry and in situ hybridization}

One or more representative tumor blocks, including mucosal areas, invasive areas of the tumor center, lateral and deep invading front, and surrounding non-neoplastic mucosa, from each patient were examined by immunohistochemistry. We performed all of the immunohistochemical analysis on the whole sections. For immunostaining of all markers except EGFR, a Dako Envision Kit (Dako Corporation, Carpinteria, CA) was used according to the manufacturer's recommendations. The antibodies and their conditions used in the present study are shown in Supplementary Table 1. 

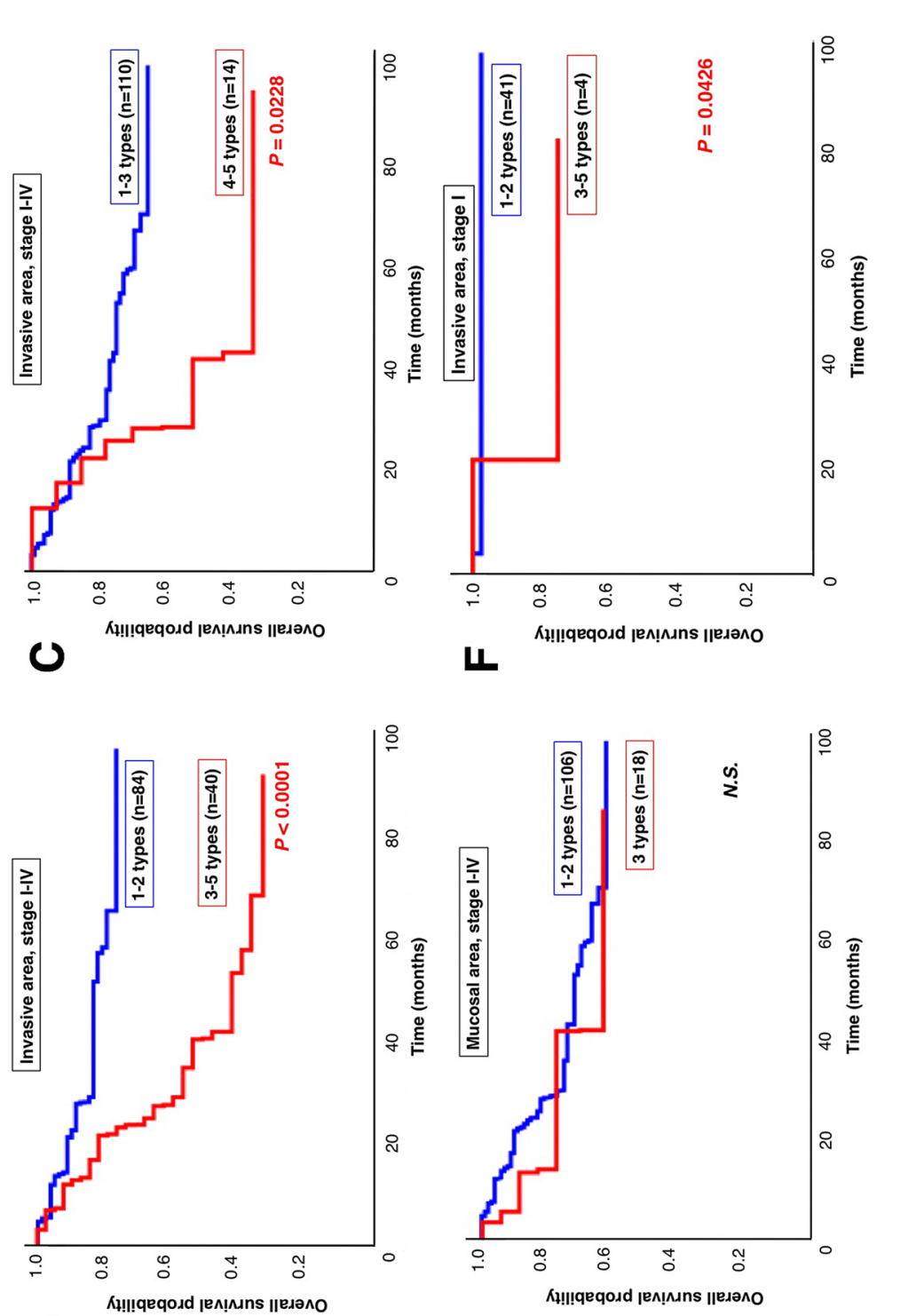

$\boldsymbol{m}$

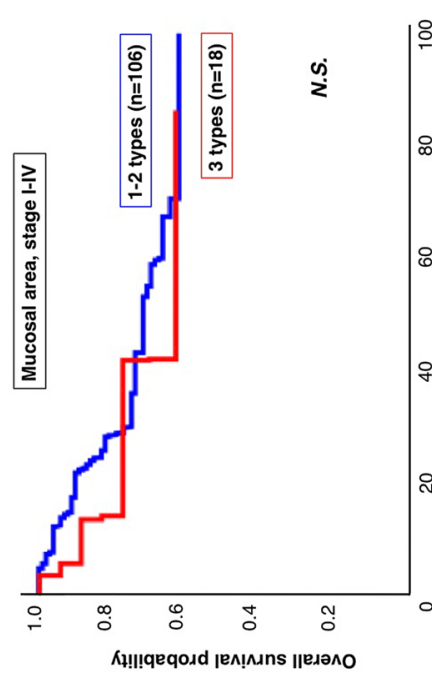

옳 올

$\because . \Xi$

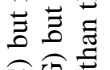

Uิ

$\leq$

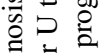

당 के

는

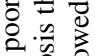

在 5

造

क

홀

क

窇

号

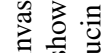

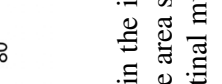

- 造高

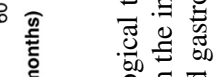

o

2
0

Q $\quad \sum_{\dot{0}}^{0} \hat{0}$

究它兘

额会营

宝定

记记

牙品

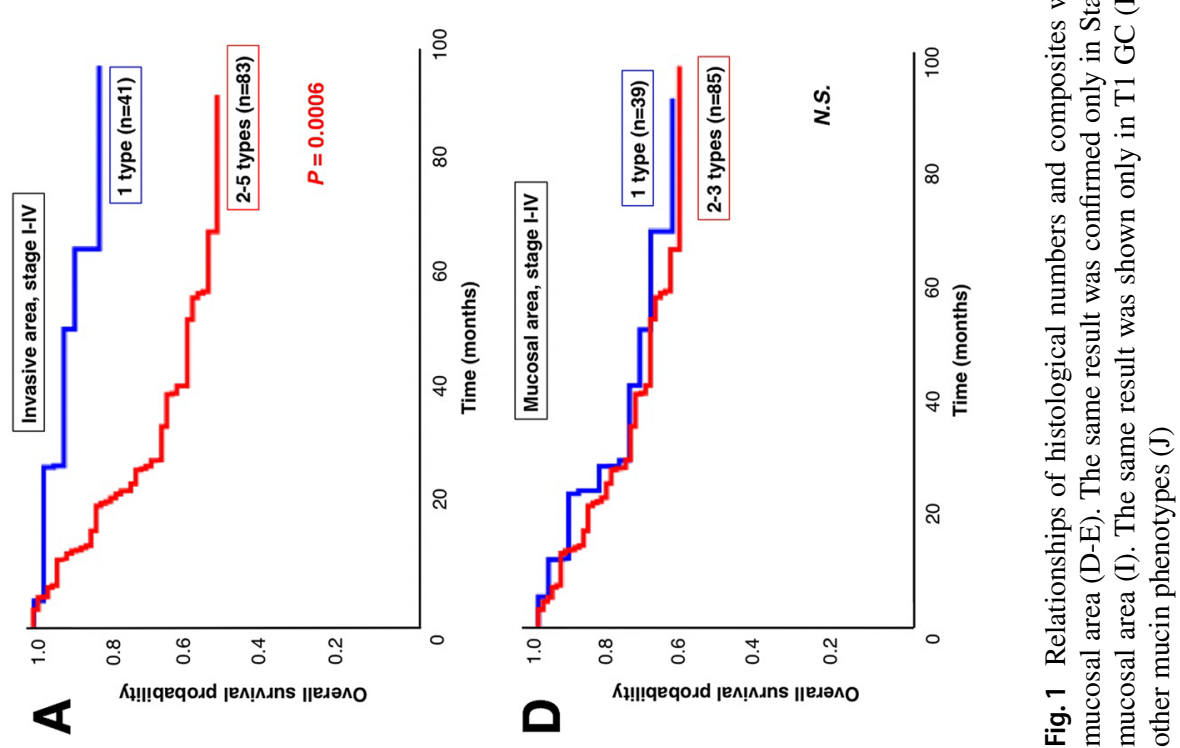



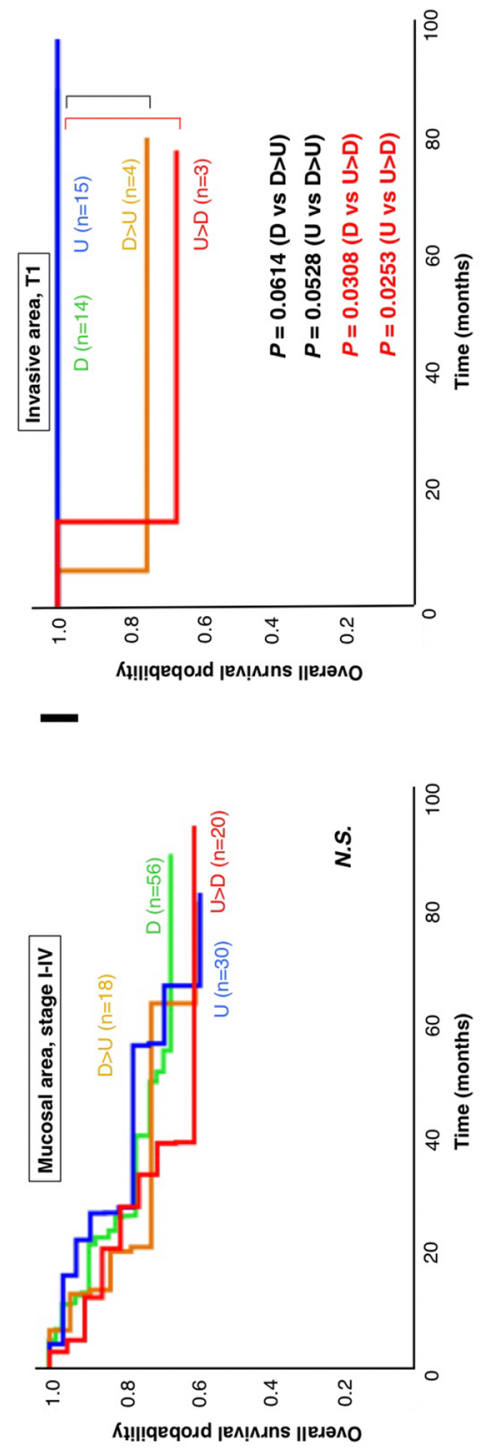

工

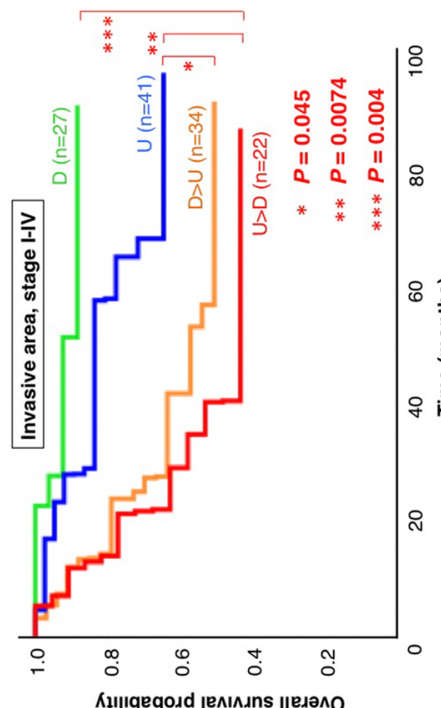

(5)

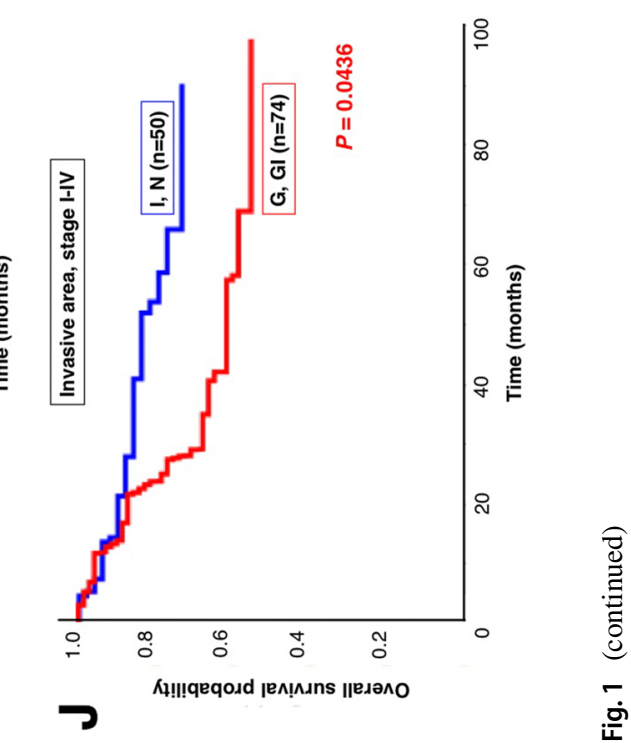


After endogenous peroxidase activity was blocked with $3 \% \mathrm{H}_{2} \mathrm{O}_{2}$-methanol for $10 \mathrm{~min}$, sections were incubated with normal goat serum (Dako Corporation) for $20 \mathrm{~min}$ to block nonspecific antibody binding sites. The sections were incubated with the primary antibodies for $1 \mathrm{~h}$ at room temperature, followed by incubations with peroxidase-labeled anti-rabbit or mouse IgG for $60 \mathrm{~min}$. For immunostaining of EGFR, a Dako EGFR pharmDx ${ }^{\mathrm{TM}}$ assay detection system (Dako Corporation) was used. Staining was completed with a 10-min incubation with the substrate-chromogen solution. The sections were counterstained with $0.1 \%$ hematoxylin. Appropriate positive and negative control samples were also stained. For Epstein-Barr virus (EBV), slides were stained on an automated slide-staining system (Benchmark XT; Ventana Medical Systems, Inc., Tucson, AZ). A ready-to-use EBER (EBV-encoded small RNA) probe (Ventana/Roche) was used together with ISH-Protease 3 pretreatment for $1 \mathrm{~h}$ and probe incubation of $28 \mathrm{~min}$.

\section{Evaluation of positive cases}

Immunostaining results were evaluated independently by 3 investigators (K. S., T. I. and G. K.), and when the evaluations differed, a decision was made by consensus, while the investigators reviewed the specimen with a multi-head microscope. Neoplastic tissue was evaluated semiquantitatively at magnifications of $\times 100$ and $\times 400$. The cutoff-point for antibody reactivity necessary to define a result as positive is shown in Supplementary Table 1. HER2 or c-MET staining was scored as previously reported [10] and was considered to be positive for overexpression if the staining was scored as $2+$ or $3+$. E-cadherin and ARID1A expression was semiquantitatively scored on a scale of 0 (no staining), $1+$ (weak), $2+$ (moderate) to $3+$ (strong; equivalent to normal epithelium or stromal cells of the stomach). A score of $2+$ or less was regarded as loss or reduction [11, 12]. MLH1 or MSH2 was considered to be a loss or reduction of nuclear reactivity together with a positive background reaction in non-neoplastic epithelial or stromal cells.

\section{Mucin phenotypes of GCs and molecular subtypes using surrogate markers}

We evaluated 124 GCs according to the criteria for classification of mucin phenotypes [13]. Furthermore, these 124 GC cases were divided into four molecular subtypes according to a previous report [14]. The microsatellite-instable (MSI) subtype was defined as tumors showing a loss or reduction of MLH1 of more than $30 \%$. Strong nuclear staining of p53 of more than $50 \%$ was used as a surrogate for the chromosomal instability (CIN) subtype, as they frequently showed a TP53 mutation [9]. In addition, overexpression of tyrosine kinase receptors such as HER2, EGFR or c-MET was used as a CIN subtype. The remaining cases showing loss or reduction of E-cadherin were categorized as genomic stability (GS). EBV subtype indicated EBER-positive tumors.
Table 1 Relationships of histological numbers in mucosal and invasive areas with clinicopathological parameters in 842 GCs

\begin{tabular}{|c|c|c|c|c|c|c|}
\hline & \multicolumn{2}{|l|}{ Invasive area } & \multirow[t]{2}{*}{$p$} & \multicolumn{2}{|l|}{ Mucosal area } & \multirow[t]{2}{*}{$p$} \\
\hline & $1-2(n=571)$ & $3-5(n=271)$ & & $1-2(n=742)$ & $\begin{array}{l}3-4 \\
(n=100)\end{array}$ & \\
\hline \multicolumn{7}{|l|}{ Age } \\
\hline$\leq 65$ & 271 & $127(32 \%)$ & NS & 342 & $56(14 \%)$ & NS \\
\hline$>65$ & 300 & $144(32 \%)$ & & 400 & $44(10 \%)$ & \\
\hline \multicolumn{7}{|l|}{ Gender } \\
\hline Male & 366 & $172(32 \%)$ & NS & 481 & $57(11 \%)$ & NS \\
\hline Female & 205 & $99(33 \%)$ & & 261 & $43(14 \%)$ & \\
\hline \multicolumn{7}{|l|}{$\mathrm{T}$ grade } \\
\hline $\mathrm{T} 1$ & 217 & $64(23 \%)$ & 0.0001 & 241 & $40(14 \%)$ & NS \\
\hline $\mathrm{T} 2-4$ & 354 & $207(37 \%)$ & & 501 & $60(11 \%)$ & \\
\hline \multicolumn{7}{|l|}{$\mathrm{N}$ grade } \\
\hline N0 & 289 & $125(30 \%)$ & NS & 367 & $47(11 \%)$ & NS \\
\hline N1-3 & 282 & $146(34 \%)$ & & 375 & $53(12 \%)$ & \\
\hline \multicolumn{7}{|l|}{$\mathbf{M}$ grade } \\
\hline M0 & 512 & $241(32 \%)$ & NS & 663 & $90(12 \%)$ & NS \\
\hline M1 & 59 & $30(34 \%)$ & & 79 & $10(11 \%)$ & \\
\hline \multicolumn{7}{|l|}{ Stage } \\
\hline I & 289 & $102(26 \%)$ & 0.0005 & 340 & $51(13 \%)$ & NS \\
\hline II-IV & 282 & $169(37 \%)$ & & 402 & $49(11 \%)$ & \\
\hline
\end{tabular}

$G C$ gastric cancer, $N S$ not significant 


\section{Statistical methods}

Analysis of clinicopathological parameters or immunohistochemical staining was performed using the Chi squared test. The Kaplan-Meier method was used to estimate the overall survival, and the difference in survival between groups was examined with a log-rank test. Univariate and multivariate Cox regression was used to evaluate the associations between clinical covariates and survival as described previously [15]. A $p$ value of $<0.05$ was considered statistically significant. The SPSS software program (SPSS Inc., Chicago, IL, USA) was used for all statistical analyses.

\section{Results}

\section{Clinicopathological characteristics associated with histological numbers}

Among the $842 \mathrm{GC}$ cases, histological components are listed in the descending order of their proportion in the mucosa and invasive areas, and the histological combination of both areas totaled 344 patterns (Supplementary Fig. 1a-c, Supplementary Table 2). The most predominant histology of mucosal areas showed a concordance with those of corresponding invasive areas except for signet-ring cell carcinoma most likely turning into poorly differentiated adenocarcinoma (Supplementary Table 3). Histological numbers ranged from 1 to 4 in the mucosal areas and from 1 to 5 in the invasive areas (Supplementary Table 4). Table 1 shows the relationship between the clinicopathological factors and histological numbers. GC cases with more histological numbers in invasive areas showed significantly higher $\mathrm{T}$ grade and staging, whereas those in mucosal areas did not show any significant associations. Furthermore, GC cases with a greater increase in histological numbers in invasive areas than mucosal areas showed significantly higher $\mathrm{T}$ grade, $\mathrm{N}$ grade, $\mathrm{M}$ grade and staging (Supplementary Table 5).

\section{Clinicopathological characteristics associated with histological composites}

The 842 GC cases were classified into four subgroups according to their histological features: a pure differentiated component with no undifferentiated component (D type); a pure undifferentiated component with no differentiated component (U type); and a histologically mixed type that consisted of both differentiated and undifferentiated components (mixed type). The histological mixed type included the differentiatedpredominant mixed type with the undifferentiated component making up less than or equal to $50 \%$ (D>U type) and the undifferentiated-predominant mixed type with the undifferentiated component making up more than 50\% (U $>$ D type).
The $842 \mathrm{GC}$ cases were divided into $445 \mathrm{D}$ type, $142 \mathrm{U}$ type, $144 \mathrm{D}>\mathrm{U}$ type and $111 \mathrm{U}>\mathrm{D}$ type in the mucosal areas, and $214 \mathrm{D}$ type, $214 \mathrm{U}$ type, $224 \mathrm{D}>\mathrm{U}$ type and $190 \mathrm{U}>\mathrm{D}$ type in the invasive areas. Characteristically, 235 (53\%) of the 445 D-type GC cases in mucosal areas displayed U type, D $>U$ type or U $>$ D type histology in the invasive areas (Supplementary Table 6). Supplementary Table 7 shows the relationship between the clinicopathological factors and histological composites. D > U type or U > D type showed higher T grade, $\mathrm{N}$ grade and staging than $\mathrm{U}$ type in the invasive areas, whereas these types were only associated with higher $\mathrm{N}$ grade in the mucosal areas (Supplementary Table 8). In addition, D $>U$ type or $U>D$ type was characteristically observed in patients aged over 65 , whereas $U$ type was more frequent in patients aged less than 65 . The male and female ratios gradually decreased significantly from $\mathrm{D}$ type to $\mathrm{U}$ type via $\mathrm{D}>\mathrm{U}$ type or $\mathrm{U}>\mathrm{D}$ type.

\section{Relationships of histological numbers and composites with patient prognosis}

We examined the relationships of histological numbers and composites with overall survival in $124 \mathrm{GC}$ cases. The prognosis of patients with more histological numbers in the invasive areas was significantly worse ( $N=1$ vs $2 \leq, p=0.0006$; $N=1-2$ vs $3 \leq, p<0.0001 ; N=1-3$ vs $4 \leq, p=0.0228$ ) (Fig. 1a-c), whereas there was no significant difference in mucosal areas (Fig. 1d-e). The same result was also confirmed only in stage I GC (Fig. 1f). Furthermore, in the 124 GC cases, the prognosis of $\mathrm{D}>\mathrm{U}$ or $\mathrm{U}>\mathrm{D}$ type (mixed type) was significantly poorer than that of $\mathrm{D}$ or $\mathrm{U}$ type (pure type) in invasive areas (Fig. 1g) but not in mucosal areas (Fig. 1h). The same result was shown only in T1 GC (Fig. 1i). Therefore, we performed the following analysis of histological composites using the classifications of mixed type and pure type. Table 2 shows the relationships of histological composites in mucosal and invasive areas with clinicopathological parameters in 842 GCs. Indeed, the results with the classifications of mixed type and pure type displayed almost the same tendency as those with four subgroups.

In a univariate analysis, more histological numbers and mixed type in invasive areas showed a significantly poorer prognosis (Table 3), but they were not independent factors in the multivariate analysis of the 124 GCs from stage I to IV. However, when the GCs were limited to stage II and III, more histological numbers were an independent factor of poor prognosis $(N=1-2$ vs $3 \leq, p=0.0325)$. 
Table 2 Relationships of histological composites in mucosal and invasive areas with clinicopathological parameters in 842 GCs

\begin{tabular}{|c|c|c|c|c|c|c|}
\hline & \multicolumn{2}{|c|}{ Invasive area } & \multirow[t]{2}{*}{$p$} & \multicolumn{2}{|c|}{ Mucosal area } & \multirow[t]{2}{*}{$p$} \\
\hline & $\begin{array}{l}\text { Pure type } \\
(n=428)\end{array}$ & Mixed type $(n=414)$ & & $\begin{array}{l}\text { Pure type } \\
(n=587)\end{array}$ & Mixed type $(n=255)$ & \\
\hline \multicolumn{7}{|l|}{ Age } \\
\hline$\leq 65$ & 230 & $184(44 \%)$ & 0.0072 & 295 & $119(36 \%)$ & NS \\
\hline$>65$ & 198 & $230(54 \%)$ & & 292 & $136(25 \%)$ & \\
\hline \multicolumn{7}{|l|}{ Gender } \\
\hline Male & 272 & $272(50 \%)$ & NS & 388 & $156(29 \%)$ & NS \\
\hline Female & 156 & $142(48 \%)$ & & 199 & $99(33 \%)$ & \\
\hline \multicolumn{7}{|l|}{$\mathrm{T}$ grade } \\
\hline $\mathrm{T} 1$ & 189 & $93(33 \%)$ & $<0.0001$ & 197 & $85(30 \%)$ & NS \\
\hline $\mathrm{T} 2-4$ & 239 & $321(57 \%)$ & & 390 & $170(30 \%)$ & \\
\hline \multicolumn{7}{|l|}{$\mathrm{N}$ grade } \\
\hline No & 267 & $145(35 \%)$ & $<0.0001$ & 309 & $103(25 \%)$ & 0.0012 \\
\hline N1-3 & 161 & $269(63 \%)$ & & 278 & $152(35 \%)$ & \\
\hline \multicolumn{7}{|l|}{$\mathbf{M}$ grade } \\
\hline M0 & 392 & $355(48 \%)$ & 0.0087 & 523 & $224(30 \%)$ & NS \\
\hline M1 & 36 & $59(62 \%)$ & & 64 & $31(33 \%)$ & \\
\hline \multicolumn{7}{|l|}{ Stage } \\
\hline I & 253 & $139(35 \%)$ & $<0.0001$ & 285 & $107(27 \%)$ & NS \\
\hline II-IV & 175 & $275(61 \%)$ & & 302 & $148(33 \%)$ & \\
\hline
\end{tabular}

$G C$ gastric cancer, $N S$ not significant

\begin{tabular}{|c|c|c|c|c|c|c|}
\hline & \multirow{2}{*}{\multicolumn{2}{|c|}{$\frac{\text { Univariate analysis }}{\mathrm{HR}(95 \% \mathrm{Cl})}$}} & \multirow[t]{2}{*}{$p$} & \multirow{2}{*}{\multicolumn{2}{|c|}{$\frac{\text { Multivariate analysis }}{\mathrm{HR}(95 \% \mathrm{Cl})}$}} & \multirow[t]{2}{*}{$p$} \\
\hline & & & & & & \\
\hline \multicolumn{7}{|l|}{ Age } \\
\hline$\leq 65(n=64)$ & 1 & Reference & NS & & & \\
\hline$>65(n=60)$ & 1.35 & $(0.73-2.52)$ & & & & \\
\hline \multicolumn{7}{|l|}{ Gender } \\
\hline Female $(n=43)$ & 1 & Reference & NS & & & \\
\hline Male $(n=81)$ & 1.39 & $(0.72-2.83)$ & & & & \\
\hline \multicolumn{7}{|l|}{ Stage } \\
\hline $\mathrm{I}(n=45)$ & 1 & Reference & $<0.0001$ & 1 & Reference & $<0.0001$ \\
\hline II-IV $(n=79)$ & 15.6 & $(4.78-95.9)$ & & 10.4 & $(2.98-65.8)$ & \\
\hline \multicolumn{7}{|l|}{ Histological numbers } \\
\hline $1-2(n=84)$ & 1 & Reference & $<0.0001$ & 1 & Reference & 0.0614 \\
\hline $3-5(n=40)$ & 3.92 & $(2.11-7.46)$ & & 1.89 & $(0.97-3.82)$ & \\
\hline \multicolumn{7}{|l|}{ Histological composites } \\
\hline Pure type $(n=68)$ & 1 & Reference & 0.0002 & 1 & Reference & NS \\
\hline Mixed type $(n=56)$ & 3.35 & $(1.17-6.7)$ & & 1.37 & $(0.68-2.91)$ & \\
\hline
\end{tabular}

$G C$ gastric cancer, $H R$ hazard ratio, $C I$ confidence interval, $N S$ not significant
Table 3 Univariate and multivariate Cox regression analysis of histological numbers and composites with overall survival in $124 \mathrm{GCs}$ invasive areas (Table 4 and Supplementary Table 1). In the 124 cases examined, molecules of each of the cancer stem cell (CSC) markers were detected as follows: CD44 in 56 (45\%) cases, CD133 in 21 (17\%) cases and ALDH1 in 78 (63\%) cases, and the expressions of these molecules were significantly related with more histological numbers or mixed type. In addition, molecules of each of the tyrosine 
Table 4 Relation of histological numbers and composites in invasive areas with cancerrelated molecules or infection status in invasive areas of 124 GCs

\begin{tabular}{|c|c|c|c|c|c|c|}
\hline \multirow[t]{2}{*}{ Invasive area } & \multicolumn{2}{|c|}{ Histological numbers } & \multirow[t]{2}{*}{$p$} & \multicolumn{2}{|c|}{ Histological composites } & \multirow[t]{2}{*}{$p$} \\
\hline & $\begin{array}{l}1-2 \\
(n=84)\end{array}$ & $\begin{array}{l}3-5 \\
(n=40)\end{array}$ & & $\begin{array}{l}\text { Pure type } \\
(n=68)\end{array}$ & Mixed type $(n=56)$ & \\
\hline \multicolumn{7}{|l|}{ CD44 } \\
\hline+ & 29 & $27(48 \%)$ & 0.0009 & 22 & $34(61 \%)$ & 0.0021 \\
\hline- & 55 & $13(19 \%)$ & & 46 & $22(32 \%)$ & \\
\hline \multicolumn{7}{|l|}{ CD133 } \\
\hline+ & 10 & $11(52 \%)$ & 0.0407 & 6 & $15(71 \%)$ & 0.0147 \\
\hline- & 74 & $29(28 \%)$ & & 62 & $41(40 \%)$ & \\
\hline \multicolumn{7}{|l|}{ ALDH1 } \\
\hline+ & 48 & $30(38 \%)$ & NS & 36 & $42(54 \%)$ & 0.0151 \\
\hline- & 36 & $10(22 \%)$ & & 32 & $14(30 \%)$ & \\
\hline \multicolumn{7}{|l|}{ HER2 } \\
\hline+ & 15 & $13(46 \%)$ & NS & 9 & $19(68 \%)$ & 0.009 \\
\hline- & 69 & $27(29 \%)$ & & 59 & $37(39 \%)$ & \\
\hline \multicolumn{7}{|l|}{ EGFR } \\
\hline+ & 13 & $11(46 \%)$ & NS & 7 & $17(71 \%)$ & 0.0061 \\
\hline- & 71 & $29(29 \%)$ & & 61 & $39(39 \%)$ & \\
\hline \multicolumn{7}{|l|}{ c-MET } \\
\hline+ & 14 & $10(42 \%)$ & NS & 6 & $18(75 \%)$ & 0.0013 \\
\hline- & 70 & $30(30 \%)$ & & 62 & $38(38 \%)$ & \\
\hline \multicolumn{7}{|l|}{ PD-L1 } \\
\hline+ & 14 & $6(30 \%)$ & NS & 9 & $11(55 \%)$ & NS \\
\hline- & 70 & $34(33 \%)$ & & 59 & $45(43 \%)$ & \\
\hline \multicolumn{7}{|l|}{ MMP7 } \\
\hline+ & 43 & $32(43 \%)$ & 0.003 & 36 & $39(52 \%)$ & NS \\
\hline- & 41 & $8(16 \%)$ & & 32 & $17(35 \%)$ & \\
\hline \multicolumn{7}{|l|}{ Laminin $5 \gamma 2$} \\
\hline+ & 27 & $20(43 \%)$ & NS & 16 & $31(66 \%)$ & 0.0004 \\
\hline- & 57 & $20(26 \%)$ & & 52 & $25(32 \%)$ & \\
\hline \multicolumn{7}{|l|}{ p53 } \\
\hline+ & 19 & $7(27 \%)$ & NS & 12 & $14(54 \%)$ & NS \\
\hline- & 65 & $33(34 \%)$ & & 56 & $42(43 \%)$ & \\
\hline \multicolumn{7}{|l|}{ CDX2 } \\
\hline+ & 32 & $17(35 \%)$ & NS & 27 & $22(45 \%)$ & NS \\
\hline- & 52 & $23(31 \%)$ & & 41 & $34(45 \%)$ & \\
\hline \multicolumn{7}{|l|}{$\beta$-catenin } \\
\hline+ & 29 & $17(37 \%)$ & NS & 26 & $20(43 \%)$ & NS \\
\hline- & 55 & $23(29 \%)$ & & 42 & $36(46 \%)$ & \\
\hline \multicolumn{7}{|l|}{ Claudin-18 } \\
\hline+ & 39 & $19(33 \%)$ & NS & 30 & $28(48 \%)$ & NS \\
\hline- & 45 & $21(32 \%)$ & & 38 & $28(42 \%)$ & \\
\hline \multicolumn{7}{|l|}{ VEGF-A } \\
\hline+ & 40 & $21(34 \%)$ & NS & 29 & $32(52 \%)$ & NS \\
\hline- & 44 & $19(30 \%)$ & & 39 & $24(38 \%)$ & \\
\hline \multicolumn{7}{|l|}{ MUC5AC } \\
\hline+ & 29 & $19(40 \%)$ & NS & 21 & $27(55 \%)$ & NS \\
\hline- & 55 & $21(28 \%)$ & & 47 & $29(38 \%)$ & \\
\hline MUC6 & & & & & & \\
\hline+ & 16 & $9(36 \%)$ & NS & 10 & $15(60 \%)$ & NS \\
\hline- & 68 & $31(31 \%)$ & & 58 & $41(41 \%)$ & \\
\hline MUC2 & & & & & & \\
\hline
\end{tabular}


Table 4 (continued)

\begin{tabular}{|c|c|c|c|c|c|c|}
\hline \multirow[t]{2}{*}{ Invasive area } & \multicolumn{2}{|c|}{ Histological numbers } & \multirow[t]{2}{*}{$p$} & \multicolumn{2}{|c|}{ Histological composites } & \multirow[t]{2}{*}{$p$} \\
\hline & $\begin{array}{l}1-2 \\
(n=84)\end{array}$ & $\begin{array}{l}3-5 \\
(n=40)\end{array}$ & & $\begin{array}{l}\text { Pure type } \\
(n=68)\end{array}$ & Mixed type $(n=56)$ & \\
\hline+ & 28 & $17(38 \%)$ & NS & 27 & $18(40 \%)$ & NS \\
\hline- & 56 & $23(29 \%)$ & & 41 & $38(48 \%)$ & \\
\hline \multicolumn{7}{|l|}{ CD10 } \\
\hline+ & 24 & $14(37 \%)$ & NS & 17 & $21(55 \%)$ & NS \\
\hline- & 60 & $26(30 \%)$ & & 51 & $35(41 \%)$ & \\
\hline \multicolumn{7}{|l|}{ Mucin phenotype } \\
\hline G, GI & 41 & $33(45 \%)$ & 0.0004 & 35 & $39(53 \%)$ & 0.045 \\
\hline $\mathrm{I}, \mathrm{N}$ & 43 & $7(14 \%)$ & & 33 & $17(34 \%)$ & \\
\hline \multicolumn{7}{|l|}{ E-cadherin } \\
\hline Retained & 47 & $24(34 \%)$ & NS & 33 & $38(54 \%)$ & 0.0444 \\
\hline Loss or reduction & 37 & $16(30 \%)$ & & 35 & $18(34 \%)$ & \\
\hline \multicolumn{7}{|l|}{ ARID1A } \\
\hline Retained & 65 & $32(33 \%)$ & NS & 55 & $42(43 \%)$ & NS \\
\hline Loss or reduction & 19 & $8(30 \%)$ & & 13 & $14(52 \%)$ & \\
\hline \multicolumn{7}{|l|}{ MLH1 } \\
\hline Retained & 74 & $37(33 \%)$ & NS & 63 & $48(43 \%)$ & NS \\
\hline Loss or reduction & 10 & $3(23 \%)$ & & 5 & $8(62 \%)$ & \\
\hline \multicolumn{7}{|l|}{ MSH2 } \\
\hline Retained & 81 & $38(32 \%)$ & NS & 67 & $52(44 \%)$ & NS \\
\hline Loss or reduction & 3 & $2(40 \%)$ & & 1 & $4(80 \%)$ & \\
\hline \multicolumn{7}{|l|}{ EBER-ISH } \\
\hline+ & 5 & $1(17 \%)$ & NS & 4 & $2(33 \%)$ & NS \\
\hline- & 79 & $39(33 \%)$ & & 64 & $54(46 \%)$ & \\
\hline \multicolumn{7}{|l|}{ H. pylori } \\
\hline+ & 21 & $19(48 \%)$ & 0.0147 & 16 & $24(60 \%)$ & 0.0331 \\
\hline - & 63 & $21(25 \%)$ & & 52 & $32(38 \%)$ & \\
\hline
\end{tabular}

$G C$ gastric cancer, $G$ gastric phenotype, $G I$ gastrointestinal phenotype, $I$ intestinal phenotype, $N$ null phenotype, $N S$ not significant

kinase receptors were detected as follows: HER2 in 28 $(23 \%)$ cases, EGFR in $24(19 \%)$ cases and c-MET in 24 $(19 \%)$ cases, and the expressions of all of these molecules were associated with mixed type. In 5 of 19 mixed-type HER2-positive GCs, HER2 expression was observed in both differentiated and undifferentiated components. Other molecules such as MMP-7, laminin $5 \gamma 2$ or E-cadherin were also associated with more histological numbers or mixed type. Furthermore, H. pylori infection was significantly associated with both more histological numbers and mixed type, whereas EBV infection did not correlate with either of them. Meanwhile, GCs with gastric mucin or mixed gastrointestinal mucin phenotype in invasive areas showed poorer prognosis than those with other mucin phenotypes ( $p=0.0436$ ) (Fig. 1j). They were also significantly associated with more histological numbers and mixed histological type, although each mucin-associated molecule was not associated with either of them.

\section{Histological characteristics and cancer-related molecules in mucosal areas predicting more histological numbers and mixed types in invasive areas}

There were significant associations between more histological numbers and mixed type in both the invasive and mucosal areas $(p<0.0001)$. More histological numbers in mucosal areas was also significantly related more histological numbers and mixed type in invasive areas. Mucosal mixed type was associated with invasive mixed type (Supplementary Table 9). Next, we analyzed cancer-related molecules that are predictive of histological diversities in invasive areas from the immunohistochemical expression of mucosal areas (Table 5). As a result, the mucosal expressions of CD44, HER2, c-MET, laminin $5 \gamma 2$ or retained E-cadherin were significantly associated with more histological numbers and mixed types in invasive areas. There were no significant 
Table 5 Relation of histological numbers and composites in invasive areas with cancerrelated molecules in mucosal areas of $124 \mathrm{GCs}$

\begin{tabular}{|c|c|c|c|c|c|c|}
\hline \multirow[t]{2}{*}{ Mucosal area } & \multicolumn{2}{|c|}{ Histological numbers } & \multirow[t]{2}{*}{$p$} & \multicolumn{2}{|c|}{ Histological composites } & \multirow[t]{2}{*}{$p$} \\
\hline & $\begin{array}{l}1-2 \\
(n=84)\end{array}$ & $\begin{array}{l}3-5 \\
(n=40)\end{array}$ & & $\begin{array}{l}\text { Pure type } \\
(n=68)\end{array}$ & Mixed type $(n=56)$ & \\
\hline \multicolumn{7}{|l|}{ CD44 } \\
\hline+ & 27 & $21(44 \%)$ & 0.0478 & 19 & $29(60 \%)$ & 0.0093 \\
\hline- & 57 & $19(25 \%)$ & & 49 & $27(36 \%)$ & \\
\hline \multicolumn{7}{|l|}{ CD133 } \\
\hline+ & 8 & $7(47 \%)$ & NS & 5 & $10(67 \%)$ & NS \\
\hline- & 76 & $33(30 \%)$ & & 63 & $46(42 \%)$ & \\
\hline \multicolumn{7}{|l|}{ ALDH1 } \\
\hline+ & 47 & $25(35 \%)$ & NS & 34 & $38(53 \%)$ & NS \\
\hline- & 37 & $15(29 \%)$ & & 34 & $18(35 \%)$ & \\
\hline \multicolumn{7}{|l|}{ HER2 } \\
\hline+ & 11 & $12(52 \%)$ & 0.0287 & 7 & $16(70 \%)$ & 0.0111 \\
\hline- & 73 & $28(28 \%)$ & & 61 & $40(40 \%)$ & \\
\hline \multicolumn{7}{|l|}{ EGFR } \\
\hline+ & 10 & $10(50 \%)$ & NS & 7 & $13(65 \%)$ & NS \\
\hline- & 74 & $30(29 \%)$ & & 61 & $43(41 \%)$ & \\
\hline \multicolumn{7}{|l|}{ c-MET } \\
\hline+ & 14 & $9(39 \%)$ & NS & 6 & $17(74 \%)$ & 0.0026 \\
\hline- & 70 & $31(31 \%)$ & & 62 & $39(39 \%)$ & \\
\hline \multicolumn{7}{|l|}{ PD-L1 } \\
\hline+ & 13 & $7(35 \%)$ & NS & 9 & $11(55 \%)$ & NS \\
\hline- & 71 & $33(32 \%)$ & & 59 & $45(43 \%)$ & \\
\hline \multicolumn{7}{|l|}{ MMP7 } \\
\hline+ & 32 & $17(35 \%)$ & NS & 28 & $21(43 \%)$ & NS \\
\hline- & 52 & $23(31 \%)$ & & 40 & $35(47 \%)$ & \\
\hline \multicolumn{7}{|l|}{ Laminin $5 \gamma 2$} \\
\hline+ & 30 & $20(40 \%)$ & NS & 17 & $33(66 \%)$ & 0.0002 \\
\hline- & 54 & $20(27 \%)$ & & 51 & $23(31 \%)$ & \\
\hline \multicolumn{7}{|l|}{ p53 } \\
\hline+ & 13 & $5(28 \%)$ & NS & 7 & $11(61 \%)$ & NS \\
\hline- & 71 & $35(33 \%)$ & & 61 & $45(42 \%)$ & \\
\hline \multicolumn{7}{|l|}{$\mathrm{CDX} 2$} \\
\hline+ & 39 & $19(33 \%)$ & NS & 34 & $24(41 \%)$ & NS \\
\hline- & 45 & $21(32 \%)$ & & 34 & $32(48 \%)$ & \\
\hline \multicolumn{7}{|l|}{$\beta$-catenin } \\
\hline+ & 23 & $11(32 \%)$ & NS & 20 & $14(41 \%)$ & NS \\
\hline- & 61 & $29(32 \%)$ & & 48 & $42(47 \%)$ & \\
\hline \multicolumn{7}{|l|}{ Claudin-18 } \\
\hline+ & 37 & $20(35 \%)$ & NS & 28 & $29(51 \%)$ & NS \\
\hline- & 47 & $20(30 \%)$ & & 40 & $27(40 \%)$ & \\
\hline \multicolumn{7}{|l|}{ VEGF-A } \\
\hline+ & 37 & $21(36 \%)$ & NS & 27 & $31(53 \%)$ & NS \\
\hline- & 47 & $19(29 \%)$ & & 41 & $25(38 \%)$ & \\
\hline \multicolumn{7}{|l|}{ MUC5AC } \\
\hline+ & 44 & $21(32 \%)$ & NS & 34 & $31(48 \%)$ & NS \\
\hline- & 40 & $19(32 \%)$ & & 34 & $25(42 \%)$ & \\
\hline MUC6 & & & & & & \\
\hline+ & 19 & $9(32 \%)$ & NS & 12 & $16(57 \%)$ & NS \\
\hline- & 65 & $31(32 \%)$ & & 56 & $40(42 \%)$ & \\
\hline MUC2 & & & & & & \\
\hline
\end{tabular}


Table 5 (continued)

\begin{tabular}{|c|c|c|c|c|c|c|}
\hline \multirow[t]{2}{*}{ Mucosal area } & \multicolumn{2}{|c|}{ Histological numbers } & \multirow[t]{2}{*}{$p$} & \multicolumn{2}{|c|}{ Histological composites } & \multirow[t]{2}{*}{$p$} \\
\hline & $\begin{array}{l}1-2 \\
(n=84)\end{array}$ & $\begin{array}{l}3-5 \\
(n=40)\end{array}$ & & $\begin{array}{l}\text { Pure type } \\
(n=68)\end{array}$ & Mixed type $(n=56)$ & \\
\hline+ & 33 & $18(35 \%)$ & NS & 31 & $20(39 \%)$ & NS \\
\hline- & 51 & $22(30 \%)$ & & 37 & $36(49 \%)$ & \\
\hline \multicolumn{7}{|l|}{ CD10 } \\
\hline+ & 26 & $13(33 \%)$ & NS & 17 & $22(56 \%)$ & NS \\
\hline- & 58 & $27(32 \%)$ & & 51 & $34(40 \%)$ & \\
\hline \multicolumn{7}{|l|}{ Mucin phenotype } \\
\hline $\mathrm{G}, \mathrm{GI}$ & 48 & $23(32 \%)$ & NS & 38 & $33(46 \%)$ & NS \\
\hline $\mathrm{I}, \mathrm{N}$ & 36 & $17(32 \%)$ & & 30 & $23(43 \%)$ & \\
\hline \multicolumn{7}{|l|}{ E-cadherin } \\
\hline Retained & 50 & $27(35 \%)$ & NS & 35 & $42(55 \%)$ & 0.0092 \\
\hline Loss or reduction & 34 & $13(28 \%)$ & & 33 & $14(30 \%)$ & \\
\hline \multicolumn{7}{|l|}{ ARID1A } \\
\hline Retained & 67 & $32(32 \%)$ & NS & 55 & $44(44 \%)$ & NS \\
\hline Loss or reduction & 17 & $8(32 \%)$ & & 13 & $12(48 \%)$ & \\
\hline \multicolumn{7}{|l|}{ MLH1 } \\
\hline Retained & 75 & $38(34 \%)$ & NS & 62 & $51(45 \%)$ & NS \\
\hline Loss or reduction & 9 & $2(18 \%)$ & & 6 & $5(45 \%)$ & \\
\hline \multicolumn{7}{|l|}{ MSH2 } \\
\hline Retained & 83 & $39(32 \%)$ & NS & 67 & $55(45 \%)$ & NS \\
\hline Loss or reduction & 1 & $1(50 \%)$ & & 1 & $1(50 \%)$ & \\
\hline
\end{tabular}

$G C$ gastric cancer, $G$ gastric phenotype, $G I$ gastrointestinal phenotype, $I$ intestinal phenotype, $N$ null phenotype, $N S$ not significant

correlations between mucosal mucin phenotypes and histological diversities in invasive areas.

\section{Molecular subtypes related with histological diversities and other cancer-related molecules}

Finally, we analyzed the molecular subtypes marking histological diversities. The $124 \mathrm{GC}$ cases were putatively classified into five molecular subtypes based on the expression pattern of surrogate markers: 51 cases $(41 \%)$ of CIN, 29 cases (23\%) of GS, 13 cases (10\%) of MSI, 6 cases (5\%) of EBV and 25 cases (20\%) of the others subtype (Table 6). The molecular subtypes of each predominant histology included poorly differentiated adenocarcinoma in GS and EBV and well- or moderately differentiated tubular adenocarcinoma in MSI. The CIN subtype of GC showed significant associations with both more histological numbers and mixed histological type, whereas the GS subtype of GC showed a significant relationship with pure type (Table 6). In the analysis of the relationship between molecular subtypes and other cancer-related molecules, CSC markers such as CD133 and ALDH1, and VEGF-A were characteristically overexpressed in CIN subtype, and conversely more infrequent in GS subtype compared with other subtypes. Loss or reduction of ARID1A or PD-L1 overexpression was frequent in the
MSI and EBV subtypes. In addition, $H$. pylori infection was observed in the CIN subtype but was infrequent in the GS subtype (Supplementary Table 10).

\section{Discussion}

In the present study, we analyzed the relationship of histological numbers and composites in GCs with clinicopathological parameters and prognosis from morphological and molecular standpoints. As predicted, GCs with more histological numbers and mixed type in invasive areas showed higher stage and poorer prognosis, which accorded with the results of previous reports $[6,16]$. In elderly patients, the differentiated type predominates in early GCs, but the histologic diversity increases with progression to advanced cancer, resulting in higher proportions of undifferentiated type [17]. In the present study, histological diversities were increased more significantly in the elderly patients aged over 65 , and the male and female ratios gradually decreased significantly from $\mathrm{D}$ type to $\mathrm{U}$ type through $\mathrm{D}>\mathrm{U}$ type and $\mathrm{U}>\mathrm{D}$ type, similar to other previous reports $[18,19]$.

Cancer is the result of the accumulation of multiple molecular abnormalities that only occur and perpetuate in long-lived and self-renewing cells such as adult stem cells 
Table 6 Relationships of molecular classification in invasive areas of 124 GCs with histological numbers and composites using surrogate markers

\begin{tabular}{|c|c|c|c|c|c|c|c|c|}
\hline & \multicolumn{8}{|c|}{ Predominant histology } \\
\hline & pap & tub1 & tub2 & por1 & por2 & sig & muc & CWLS \\
\hline CIN $(n=51)$ & $11(22 \%)$ & $13(27 \%)$ & $10(20 \%)$ & $3(6 \%)$ & $12(24 \%)$ & 0 & $2(4 \%)$ & 0 \\
\hline GS $(n=29)$ & 0 & 0 & 0 & 0 & $24(83 \%)$ & $2(7 \%)$ & $3(10 \%)$ & 0 \\
\hline $\operatorname{MSI}(n=13)$ & $1(8 \%)$ & $3(23 \%)$ & $7(54 \%)$ & $1(8 \%)$ & $1(8 \%)$ & 0 & 0 & 0 \\
\hline $\operatorname{EBV}(n=6)$ & 0 & $1(17 \%)$ & $1(17 \%)$ & $1(17 \%)$ & $2(33 \%)$ & 0 & 0 & $1(17 \%)$ \\
\hline \multirow[t]{3}{*}{ Others $(n=25)$} & $2(8 \%)$ & $5(20 \%)$ & $6(24 \%)$ & $1(4 \%)$ & $11(44 \%)$ & 0 & 0 & 0 \\
\hline & \multicolumn{4}{|c|}{ Histological numbers } & \multicolumn{4}{|c|}{ Histological composites } \\
\hline & \multicolumn{2}{|l|}{$1-2(n=84)$} & $3-5(n=40)$ & $p$ & Pure type $(n=68)$ & \multicolumn{2}{|c|}{ Mixed type $(n=56)$} & $p$ \\
\hline $\mathrm{CIN}(n=51)$ & 29 & \multicolumn{2}{|c|}{$22(43 \%)$} & 0.03 & 20 & \multicolumn{2}{|c|}{$31(61 \%)$} & 0.0035 \\
\hline GS $(n=29)$ & 19 & \multicolumn{2}{|c|}{$10(34 \%)$} & NS & 23 & \multicolumn{2}{|c|}{$6(21 \%)$} & 0.0025 \\
\hline $\operatorname{MSI}(n=13)$ & 10 & \multicolumn{2}{|c|}{$3(23 \%)$} & NS & 5 & \multicolumn{2}{|c|}{$8(61 \%)$} & NS \\
\hline $\operatorname{EBV}(n=6)$ & 5 & \multicolumn{2}{|c|}{$1(17 \%)$} & NS & 4 & \multicolumn{2}{|c|}{$2(33 \%)$} & NS \\
\hline Others $(n=25)$ & 21 & \multicolumn{2}{|c|}{$4(16 \%)$} & NS & 16 & \multicolumn{2}{|c|}{$9(36 \%)$} & NS \\
\hline
\end{tabular}

$G C$ gastric cancer, pap papillary adenocarcinoma, tub tubular adenocarcinoma, por poorly differentiated adenocarcinoma, sig signet-ring cell carcinoma, тис mucinous adenocarcinoma, $C W L S$ carcinoma with lymphoid stroma, CIN chromosomal-instable, GS genomically stable, $M S I$ microsatellite-instable, $E B V$ Epstein-Barr virus, $N S$ not significant

[20]. A previous study in mice revealed that the induction of genetic or epigenetic events specifically in gastric stem cells can lead to the development of GCs [21]. This malignant transformation of gastric stem cells also occurs as a result of $H$. pylori infection and CD44 induction [22-24]. In the present study, the expression of CSC markers and $H$. pylori infection were significantly associated with more histological numbers and mixed type in invasive areas. Previous studies showed that several GC-related molecules could be useful as an adjunct to TNM staging [25, 26]. However, GC heterogeneity appears to have had a negative impact on the response to therapies against HER2, FGFR and EGFR and potentially CCND1 and MYC [27]. The present study showed that overexpressions of tyrosine kinase receptors such as HER2, EGFR or c-MET were all associated with mixed type in invasive areas. The extent of the heterogeneity of HER 2 expression within primary GCs varied widely across studies, from 5 to $69 \%$, and those between primary GCs and metastatic sites also varied [8]. Regarding the mixed type of GCs, some authors reported that HER 2 expression was restricted to the intestinal component [28], whereas others observed HER2 expression in both intestinal and diffuse components, supporting the hypothesis of clonal origin [29]. The present study also showed that HER2 expression was observed in both components. Several reports disclosed that the two components of the mixed type of GCs displayed similar DNA copy changes and $\mathrm{CpG}$ island hypermethylation status [30, 31] and had a higher number of methylated genes than pure diffuse or intestinal GCs [31]. These results indicate that the mixed type of GCs might emerge from a clonal origin.

The discrepancies between endoscopic biopsy and postoperative histology range from 25 to $53 \%$, probably because the endoscopic biopsy samples only a small amount of the cancerous lesion, and there is much difference in tumor microenvironment interaction between mucosal and invasive areas [32-35]. Previous reports showed that HER2 expression is more frequent in the luminal tumor layers rather than deeper ones [36] and that HER2 assessment in biopsy tissues might predict the HER2 status of the whole tumor [37]. In addition to HER2, we showed that CD44, c-MET, laminin $5 \gamma 2$ or retained E-cadherin were also useful markers predicting more histological diversities in invasive areas from endoscopic biopsy specimens. CD44 and c-MET are also the target molecules for gastric tumor-initiating cell-targeted therapeutic strategies, and their expressions are associated with chemoresistance and poor prognosis [35].

Genomic instability has often been proposed as a major driver of intratumoral heterogeneity and as a potential marker of its extent. Raynaud et al. reported that tumors with the greatest intratumoral heterogeneity exhibited high numbers of both clonal and subclonal mutations, suggesting that genomic instability emerged early and was sustained and fostered during tumor evolution [38]. They also revealed that this type of molecular phenotype was common in the CIN subtype of GC and in cancers of the lung, bladder and head and neck, and skin melanoma [38]. In the present study, the CIN subtype showed significant associations with both more 
histological numbers and mixed histological type, whereas the GS subtype showed a significant relationship with pure type. Loss or reduction of ARID1A and PD-L1 expression was frequently observed in the MSI and EBV subtypes, similar to the results of a previous report [39].

There is very limited information on the nature and the mechanisms of intratumor morphological heterogeneity in human cancers. Epithelial-mesenchymal transition (EMT) is reported to induce a variety of malignant features in cancer cells, such as cancer stemness, chemoresistance, immune evasion, altered metabolism, and blocked senescence [40]. EMT and CSC were reported to be involved in the role of morphological diversity of invasive breast carcinomas [41]. The changes in cell adhesion occurring within EMT might lead to morphologically different architectural arrangements of tumor cells. In the present study, we showed the relationship of histological diversities such as histological numbers and composites with clinicopathological significance and identified characteristic molecules marking these histological diversities. Our study is limited by its retrospective design and the small number of cases with each tumor molecular subtype. We hope that the present data can contribute to the early diagnosis and prevention, and effective treatment of GC.

Funding This work was supported by Grants-in-Aid for Scientific Research (19K07434) from the Japan Society for the Promotion of Science. This work was also supported by the Takeda Science Foundation.

\section{Compliance with ethical standards}

Conflict of interest The authors declare no conflicts of interest in association with the present study.

\section{References}

1. Zheng HC, Zheng YS, Xia P, Xu XY, Xing YN, Takahashi H, et al. The pathobiological behaviors and prognosis associated with Japanese gastric adenocarcinomas of pure WHO histological subtypes. Histol Histopathol. 2010;25:445-52.

2. Adachi Y, Yasuda K, Inomata M, Sato K, Shiraishi N, Kitano S. Pathology and prognosis of gastric carcinoma: well versus poorly differentiated type. Cancer. 2000;89:1418-24.

3. Luinetti O, Fiocca R, Villani L, Alberizzi P, Ranzani GN, Solcia E. Genetic pattern, histological structure, and cellular phenotype in early and advanced gastric cancers: evidence for structurerelated genetic subsets and for loss of glandular structure during progression of some tumors. Hum Pathol. 1998;29:702-9.

4. Ohkura Y. Histological diversity of early gastric carcinoma. The diversity of gastric carcinoma: pathogenesis, diagnosis and therapy. Tokyo: Springer; 2005. p. 117-25.

5. Tamura G. Promoter methylation status of tumor suppressor and tumor-related genes in neoplastic and non-neoplastic gastric epithelia. Histol Histopathol. 2004;19:221-8.

6. Takizawa K, Ono H, Kakushima N, Tanaka M, Hasuike N, Matsubayashi $\mathrm{H}$, et al. Risk of lymph node metastases from intramucosal gastric cancer in relation to histological types: how to manage the mixed histological type for endoscopic submucosal dissection. Gastric Cancer. 2013;16:531-6.

7. Lee HW, Kim K. Acquisition of histologic diversity contributes to not only invasiveness but also lymph node metastasis in early gastric cancer. Pathol Res Pract. 2017;213:1023-8.

8. Gullo I, Carneiro F, Oliveira C, Almeida GM. Heterogeneity in gastric cancer: from pure morphology to molecular classifications. Pathobiology. 2018;85:50-63.

9. Cancer Genome Atlas Research Network. Comprehensive molecular characterization of gastric adenocarcinoma. Nature. 2014;513:202-9.

10. Wang M, Liang L, Lei X, Multani A, Meric-Bernstam F, Tripathy $\mathrm{D}$, et al. Evaluation of cMET aberration by immunohistochemistry and fluorescence in situ hybridization (FISH) in triple negative breast cancers. Ann Diagn Pathol. 2018;35:69-76.

11. Ma YR, Siegal GP, Wei S. Reacquisition of E-cadherin expression in metastatic deposits of signet-ring cell carcinoma of the upper gastrointestinal system: a potential anchor for metastatic deposition. J Clin Pathol. 2017;70:528-32.

12. Ashizawa M, Saito M, Min AKT, Ujiie D, Saito K, Sato T, et al. Prognostic role of ARID1A negative expression in gastric cancer. Sci Rep. 2019;9:6769.

13. Sentani K, Matsuda M, Oue N, Uraoka N, Naito Y, Sakamoto $\mathrm{N}$, et al. Clinicopathological significance of MMP-7, laminin gamma2 and EGFR expression at the invasive front of gastric carcinoma. Gastric Cancer. 2014;17:412-22.

14. Gonzalez RS, Messing S, Tu X, McMahon LA, Whitney-Miller CL. Immunohistochemistry as a surrogate for molecular subtyping of gastric adenocarcinoma. Hum Pathol. 2016;56:16-21.

15. Hattori T, Sentani K, Naohide O, Sakamoto N, Yasui W. Clinicopathological significance of SPC18 in colorectal cancer: SPC18 participates in tumor progression. Cancer Sci. 2017;108:143-50.

16. Horiuchi Y, Fujisaki J, Yamamoto N, Ishizuka N, Omae M, Ishiyama A, et al. Undifferentiated-type component mixed with differentiated-type early gastric cancer is a significant risk factor for endoscopic non-curative resection. Dig Endosc. 2018;30:624-32.

17. Inoshita $\mathrm{N}$, Yanagisawa $\mathrm{A}$, Arai $\mathrm{T}$, Kitagawa $\mathrm{T}$, Hirokawa $\mathrm{K}$, Kato Y. Pathological characteristics of gastric carcinomas in the very old. Jpn J Cancer Res. 1998;89:1087-92.

18. Arai T, Takubo K. Clinicopathological and molecular characteristics of gastric and colorectal carcinomas in the elderly. Pathol Int. 2007;57:303-14.

19. Zheng HC, Li XH, Hara T, Masuda S, Yang XH, Guan YF, et al. Mixed-type gastric carcinomas exhibit more aggressive features and indicate the histogenesis of carcinomas. Virchows Arch. 2008;452:525-34.

20. Blokzijl F, de Ligt J, Jager M, Sasselli V, Roerink S, Sasaki N, et al. Tissue-specific mutation accumulation in human adult stem cells during life. Nature. 2016;538:260-4.

21. Barker N, Huch M, Kujala P, van de Wetering M, Snippert HJ, van Es JH, et al. Lgr5(+ve) stem cells drive self-renewal in the stomach and build long-lived gastric units in vitro. Cell Stem Cell. 2010;6:25-36

22. Giannakis M, Chen SL, Karam SM, Engstrand L, Gordon JI. Helicobacter pylori evolution during progression from chronic atrophic gastritis to gastric cancer and its impact on gastric stem cells. Proc Natl Acad Sci USA. 2008;105:4358-63.

23. Sigal M, Rothenberg ME, Logan CY, Lee JY, Honaker RW, Cooper RL, et al. Helicobacter pylori activates and expands Lgr5(+) stem cells through direct colonization of the gastric glands. Gastroenterology. 2015;148(1392-1404):e1321.

24. Choi YJ, Kim N, Chang H, Lee HS, Park SM, Park JH, et al. Helicobacter pylori-induced epithelial-mesenchymal transition, a potential role of gastric cancer initiation and an emergence of stem cells. Carcinogenesis. 2015;36:553-63. 
25. Lin X, Zhao Y, Song WM, Zhang B. Molecular classification and prediction in gastric cancer. Comput Struct Biotechnol J. 2015;13:448-58.

26. Sawada T, Yashiro M, Sentani K, Oue N, Yasui W, Miyazaki $\mathrm{K}$, et al. New molecular staging with $\mathrm{G}$-factor supplements TNM classification in gastric cancer: a multicenter collaborative research by the Japan Society for Gastroenterological Carcinogenesis G-Project committee. Gastric Cancer. 2015;18:119-28.

27. Alsina M, Gullo I, Carneiro F. Intratumoral heterogeneity in gastric cancer: a new challenge to face. Ann Oncol. 2017;28:912-3.

28. Kataoka Y, Okabe H, Yoshizawa A, Minamiguchi S, Yoshimura $\mathrm{K}$, Haga $\mathrm{H}$, et al. HER2 expression and its clinicopathological features in resectable gastric cancer. Gastric Cancer. 2013;16:84-93.

29. Barros-Silva JD, Leitão D, Afonso L, Vieira J, Dinis-Ribeiro M, Fragoso M, et al. Association of ERBB2 gene status with histopathological parameters and disease-specific survival in gastric carcinoma patients. Br J Cancer. 2009;100:487-93.

30. Carvalho B, Buffart TE, Reis RM, Mons T, Moutinho C, Silva P, et al. Mixed gastric carcinomas show similar chromosomal aberrations in both their diffuse and glandular components. Cell Oncol. 2006;28:283-94.

31. Park SY, Kook MC, Kim YW, Cho NY, Kim TY, Kang GH. Mixed-type gastric cancer and its association with high-frequency CpG island hypermethylation. Virchows Arch. 2010;456:625-33.

32. Palli D, Bianchi S, Cipriani F, Duca P, Amorosi A, Avellini C, et al. Reproducibility of histologic classification of gastric cancer. Br J Cancer. 1991;63:765-8.

33. Namieno T, Koito K, Higashi T, Shimamura T, Yamashita K, Sato $\mathrm{N}$, et al. Assessing the suitability of gastric carcinoma for limited resection: histologic differentiation of endoscopic biopsy. World J Surg. 1998;22:865-8.

34. Jeon SR, Cho WY, Jin SY, Cheon YK, Choi SR, Cho JY. Optical biopsies by confocal endomicroscopy prevent additive endoscopic biopsies before endoscopic submucosal dissection in gastric epithelial neoplasias: a prospective, comparative study. Gastrointest Endosc. 2011;74:772-80.

35. Gao JP, Xu W, Liu WT, Yan M, Zhu ZG. Tumor heterogeneity of gastric cancer: from the perspective of tumor-initiating cell. World J Gastroenterol. 2018;24:2567-81.

36. Tominaga N, Gotoda T, Hara M, Hale MD, Tsuchiya T, Matsubayashi J, et al. Five biopsy specimens from the proximal part of the tumor reliably determine HER2 protein expression status in gastric cancer. Gastric Cancer. 2016;19:553-60.

37. Kanayama K, Imai H, Yoneda M, Hirokawa YS, Shiraishi T. Significant intratumoral heterogeneity of human epidermal growth factor receptor 2 status in gastric cancer: a comparative study of immunohistochemistry, FISH, and dual-color in situ hybridization. Cancer Sci. 2016;107:536-42.

38. Raynaud F, Mina M, Tavernari D, Ciriello G. Pan-cancer inference of intra-tumor heterogeneity reveals associations with different forms of genomic instability. PLoS Genet. 2018;14:e1007669.

39. Kim YB, Ahn JM, Bae WJ, Sung CO, Lee D. Functional loss of ARID1A is tightly associated with high PD-L1 expression in gastric cancer. Int J Cancer. 2019;145:916-26.

40. Lu W, Kang Y. Epithelial-mesenchymal plasticity in cancer progression and metastasis. Dev Cell. 2019;49:361-74.

41. Denisov EV, Skryabin NA, Gerashchenko TS, Tashireva LA, Wilhelm J, Buldakov MA, et al. Clinically relevant morphological structures in breast cancer represent transcriptionally distinct tumor cell populations with varied degrees of epithelial-mesenchymal transition and CD44 + CD24- stemness. Oncotarget. 2017;8:61163-80.

Publisher's Note Springer Nature remains neutral with regard to jurisdictional claims in published maps and institutional affiliations. 